\title{
Oxygen-Accelerated Photopolymerization of Methyl Methacrylate in the Presence of Triethylamine
}

\author{
Rikiya Sato, Tomoki Kurihara, and Makoto TaKeIshi \\ Department of Materials Science and Engineering, Yamagata University, \\ Yonezawa, Yamagata 992, Japan
}

(Received July 22, 1997)

\begin{abstract}
KEY WORDS Methyl Methacrylate / Photopolymerization / Triethylamine / Oxygen / Charge-Transfer Complex /
\end{abstract}

We reported the oxygen-accelerated photopolymerization of methyl methacrylate (MMA) in the presence of amides $^{1}$ and ethers, ${ }^{2}$ in which oxygen forms chargetransfer complexes with additives that decompose under photoirradiation generating radical species to initiate the polymerization. Similar acceleration by oxygen was previously observed in a photopolymerization of styrene. ${ }^{3}$ Since oxygen usually inhibits the radical polymerization of vinyl monomers, little attention has been given to the effects of oxygen on the photopolymerization of vinyl monomers. However, electron-accepting oxygen is known to form charge-transfer (C-T) complexes with electron-donating molecules such as amines, ${ }^{4,5}$ and photoreactions of the complexes were investigated. ${ }^{6}$

Amines can by themselves accelerate the photopolymerization of vinyl monomers in the absence of oxygen. ${ }^{7,8}$ On the basis of experimental data, an excited monomer was found to form complexes with amines, the decomposition of which leads to initiation of radical polymerization. In the course of our study on the photopolymerization of vinyl monomers under oxygen, electron-donating triethylamine (TEA) was found to accelerate the polymerization of MMA. This paper describes the mechanism of oxygen-accelerated photopolymerization.

\section{EXPERIMENTAL}

\section{Materials}

MMA was purified by distillation under reduced pressure after the usual treatment. TEA was purified by distillation after drying over potassium hydroxide. Other reagent-grade materials were used without further purification.

\section{Polymerization}

A magnetic stirring bar and monomer were sealed in a Pyrex ampule, with or without the amine, under nitrogen or oxygen after the contents had been degassed by repeated freezing and thawing. The ampule was placed vertically in a Pyrex cooling jacket thermostated at $14^{\circ} \mathrm{C}$ by circulating water. The polymerization was carried out by irradiating with a $400-\mathrm{W}$ high-pressure mercury lamp (Riko UVL-400HA). The distance from the jacket to the lamp was $5 \mathrm{~cm}$. After a certain photoirradiation time, the reaction mixture was poured into methanol. The precipitated polymer was separated by filtration and dried in vacuo at $60^{\circ} \mathrm{C}$ to constant weight. The conversion was determined gravimetrically to calculate the rate of polymerization.

\section{UV Spectra}

UV spectra were obtained on a Hitachi 100-60 spectrometer, as reported earlier. ${ }^{1}$

\section{Measurement of Molecular Weight}

Number-average molecular weights $\left(\bar{M}_{n}\right)$ of polyMMA were calculated from the intrinsic viscosities determined in benzene at $30^{\circ} \mathrm{C}$ by

$$
[\eta]=8.69 \times 10^{-5} \times \bar{M}_{n}^{0.769}
$$

\section{RESULTS AND DISCUSSION}

The results of the photopolymerization of MMA in the presence of TEA are presented in Table I. TEA enhanced the polymerization under nitrogen (runs 1 and 3 ), though the monomer was diluted with the amine. This indicates the generation of initiating radicals. The photopolymerization of MMA in the presence of TEA has been reported in which the initiating radicals were assumed generated by decomposition of a complex formed with excited MMA and TEA. ${ }^{7}$ Accordingly, rate enhancement by TEA in this photopolymerization is considered induced in a similar manner. A possible mechanism of the reaction is as follows: 1) the excited MMA and TEA form a C-T complex, 2) an electron transfer from the amine to MMA occurs in the C-T complex yielding a radical cation and radical anion, 3) proton transfer from the radical cation to the radical anion generates radical species, and one or both of which initiate the polymerization (Scheme 1).

Table I. Photopolymerization of MMA in the presence of TEA ${ }^{\mathrm{a}}$

\begin{tabular}{cccc}
\hline Run & Amine & Atmosphere & Conversion/\% \\
\hline 1 & None $^{\mathrm{b}}$ & $\mathrm{N}_{2}$ & 4.0 \\
2 & None $^{\mathrm{b}}$ & $\mathrm{O}_{2}$ & 0 \\
3 & TEA & $\mathrm{N}_{2}$ & 16.3 \\
4 & TEA & $\mathrm{O}_{2}$ & 31.5 \\
\hline
\end{tabular}

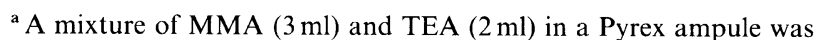
irradiated for $4 \mathrm{~h}$ at $14^{\circ} \mathrm{C}$. ${ }^{\text {b }} \mathrm{MMA}(5 \mathrm{ml})$ was photopolymerized in bulk. 


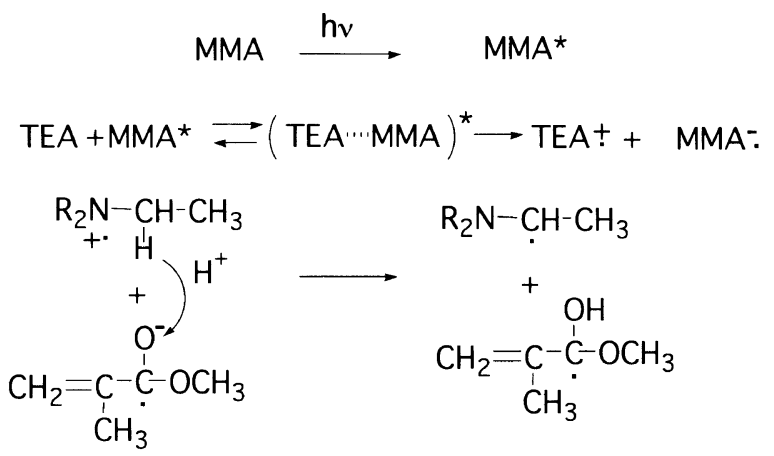

Scheme 1.

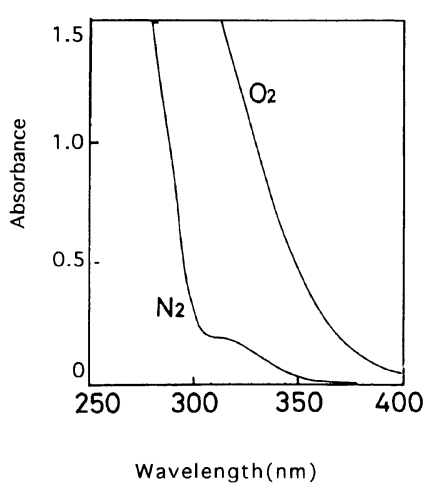

Figure 1. UV absorption spectra of TEA under $\mathrm{N}_{2}$ and $\mathrm{O}_{2}$.

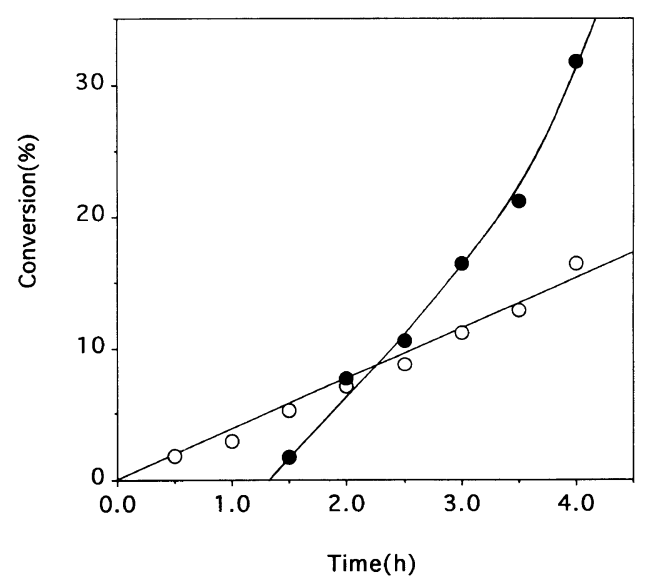

Figure 2. Photopolymerization of MMA in the presence of TEA under $\mathrm{N}_{2}(\mathrm{O})$ and $\mathrm{O}_{2}(\bigcirc)$. MMA $3 \mathrm{ml}$; TEA $2 \mathrm{ml}$.

In usual cases, oxygen inhibits radical polymerization of vinyl monomers, whereas, in some cases, a little oxygen is incorporated in the polymer backbones by copolymerization to form a peroxide structure. ${ }^{10}$ As seen in Table I, although the polymerization of MMA was effectively inhibited by oxygen in the absence of TEA, conversion was greater under oxygen than nitrogen in the presence of the amine (runs 2 and 4). Oxygen forms C-T complexes with electron donors. ${ }^{6}$ Therefore, our previous finding that the photopolymerization of MMA is enhanced under oxygen in the presence of amides ${ }^{1}$ or ethers ${ }^{2}$ as electron donors is discussed in terms of the formation of C-T complexes which decompose photochemically yielding initiating radicals. The electron-donating amines form C-T complexes with oxygen; hence, UV spectra of the TEA were measured under oxygen to examine the

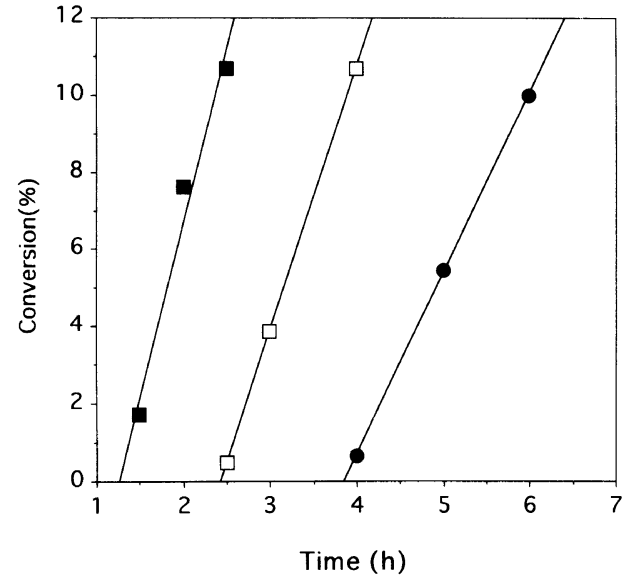

Figure 3. Effects of TEA concentration on the photopolymerization of MMA under oxygen. MMA 5.6 M; TEA: 1.44 M ( $), 2.00 \mathrm{M}(\square)$, $2.88 \mathrm{M}(\square)$ in benzene under oxygen.

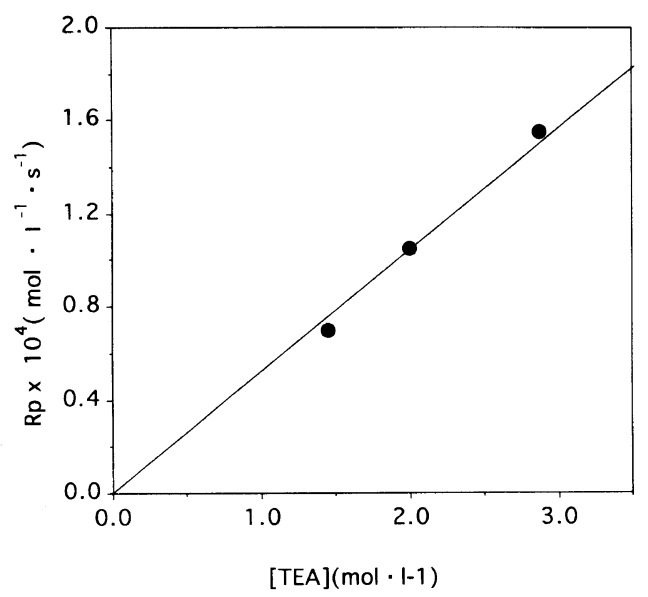

Figure 4. Plot of rate of the photopolymerization of MMA against TEA concentration. The data were taken from Figure 3.

formation of the complexes. First, nitrogen was bubbled into the amine in a quartz cell and the UV absorption spectrum was recorded. Next, oxygen was bubbled into the amine to see the change in the absorption. As shown in Figure 1, extra absorption was observed under oxygen as reported by Stenberg et al. ${ }^{11}$ This extra absorption band due to charge-transfer interaction disappeared reversibly on bubbling nitrogen again. Complex formation can be ascribed to contact or collisional interactions. $^{5}$

Figure 2 shows the effects of oxygen on the photopolymerization of MMA in the presence of TEA. Induction was observed under oxygen, and the rate of the polymerization after the period was greater than under nitrogen. This time-conversion profile of the polymerization with TEA is very similar to that previously observed for the photo-induced polymerization using ethers, ${ }^{2}$ suggesting a mechanistic similarity. The induction period caused by oxygen is due to the usual inhibition.

Figure 3 shows the photopolymerization of MMA under oxygen at various concentrations of TEA. At higher TEA concentrations, the induction period was shortened and the polymerization rate was greater. The dependence of the rate after induction period on TEA concentration showed a linear relationship (Figure 4). 


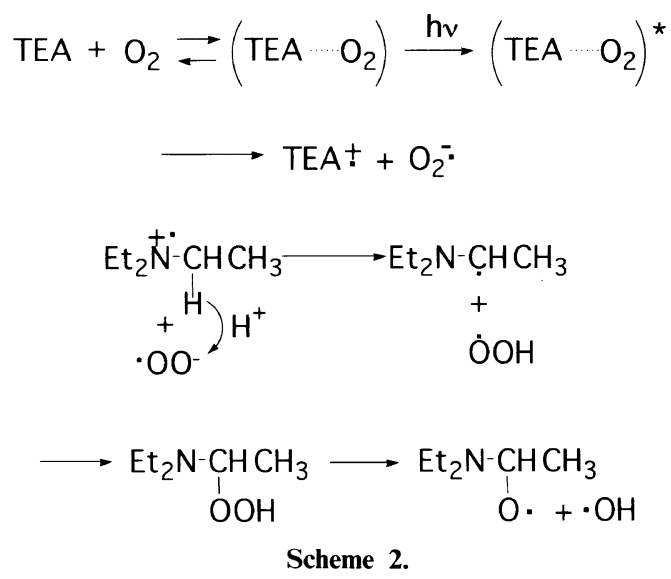

Scheme 2.

Table II. Effects of oxygen on the molecular weight of poly(MMA)

\begin{tabular}{cccc}
\hline Run & Atmosphere & Conversion $/ \%$ & $\bar{M}_{n} \times 10^{-4 \mathrm{~b}}$ \\
\hline 1 & $\mathrm{~N}_{2}$ & 7.0 & 7.50 \\
2 & $\mathrm{O}_{2}$ & 7.7 & 1.66 \\
\hline
\end{tabular}

a Photopolymerization was carried out with $3 \mathrm{ml}$ MMA and $2 \mathrm{ml}$ TEA for $2 \mathrm{~h}$ at $14^{\circ} \mathrm{C}$. ${ }^{b}$ The number- average molecular weight was calculated based on viscometry. ${ }^{10}$

On the basis of these results and the complex formation of TEA with oxygen reported by Tsubomura et al., ${ }^{6 \mathrm{~b}}$ we propose the following mechanism for the oxygenaccelerated photopolymerization of MMA in the presence of TEA: 1) TEA forms a C-T complex with oxygen, 2) oxygen accepts an electron from the TEA in the excited $\mathrm{C}$ - $\mathrm{T}$ complex to generate a radical anion of oxygen, 3 ) hydroperoxide is formed via a proton transfer, and 4) photo-induced decomposition of the hydroperoxide generates radical species to initiate the polymeri- zation (Scheme 2). If polymerization proceeds via this mechanism, the molecular weight of the polymer obtained should decrease in the presence of oxygen, because the number of initiating radicals increases. As shown in Table II, the molecular weight of the polymer obtained under oxygen decreased with increase in conversion.

The more effective acceleration by oxygen was observed with other amines such as $N, N$-dimethylbenzylamine, and further investigation to elucidate the mechanism is now in progress.

\section{REFERENCES}

1. a) M. Takeishi and G.-Y. Tao, J. Poly. Sci., Part C, Polym. Lett. Ed., 27, 301 (1989). b) M. Takeishi and G.-Y. Tao, J. Macromol. Sci., Chem., A27, 87 (1990).

2. T. Kurihara, R. Sato, and M. Takeishi, Polym. J., 23, 1397 (1991).

3. T. Kodaira, K. Hayashi, and T. Ohnishi, Polym. J., 4, 1 (1973).

4. a) D. E. Evance, J. Chem. Soc., 345 (1953); ibid., 1351 (1957); ibid., 3885 (1957); ibid., 2753 (1959); ibid., 1735 (1960); ibid., 1987 (1961). b) N. Kulevsky, C. Niu, and V. I. Stenberg, J. Org. Chem., 38, 1154 (1973).

5. H. Tsubomura and R. S. Mulliken, J. Am. Chem. Soc., 82, 5966 (1960).

6. a) R. F. Bartholomew and R. S. Davidson, J. Chem. Soc., (C), 2342 (1971). b) H. Tsubomura, T. Yagishita, and H. Toi, Bull. Chem. Soc. Jpn., 46, 3051 (1973). c) K. Maeda, A. Nakane, and H. Tsubomura, Bull. Chem. Soc. Jpn., 48, 2448 (1975). d) M. Tamres and R. L. Strong, in "Molecular Association," Vol. 2 R. Foster, Ed., Academic, London, 1979, p 397.

7. K. Yokota, T. Tomioka, Y. Ono, and K. Kuno, J. Polym. Sci., $A-1,10,1335$ (1972).

8. J. Barton, I. Leboc, I. Capek, and J. Tkac, Makromol. Chem., Rapid Commun., 1, 7 (1980).

9. T. G. Fox, J. B. Kinsinger, H. F. Mason, and E. M. Schnele, Polym., 3, 71 (1962).

10. a) G. V. Schulz and G. Henrici, Makromol. Chem., 18, 437 (1956). b) C. H. Bamford and P. R. Morris, Makromol. Chem., 87, 73 (1965)

11. V. I. Stenberg, R. D. Olson, C. T. Wang, and N. Kulvessky, J. Org. Chem., 32, 3227 (1967). 